\title{
Producción de variedades de poroto en dos localidades del Chaco Central
}

\author{
Cowpea varieties production in two Central Chaco locations
}

\author{
Cipriano Ramón Enciso Garay ${ }^{*}{ }^{*}$, Cesar Caballero Mendoza ${ }^{1}$, Jorge Daniel González ${ }^{1}$, \\ Jenny Dueck Toews ${ }^{2}$, José Maria González Balbuena ${ }^{2}$, Victoria Rossmary Santacruz Oviedo ${ }^{1}$ \\ y Fanni Ruiz ${ }^{1}$
}

${ }^{1}$ Facultad de Ciencias Agrarias, Universidad Nacional de Asunción. San Lorenzo, Paraguay.

${ }^{2}$ Cooperativa Chortitzer Ltda. Loma Plata, Paraguay.

* Autor para correspondencia (cenciso@agr.una.py)

Recibido: 21/05/2015; Aceptado: 15/06/2015. http://dx.doi.org/10.18004/investig.agrar.2015.junio.18-26

\section{RESUMEN}

El poroto es una de las leguminosas más consumida en el Paraguay, considerado como rubro de autoconsumo y renta de la agricultura familiar. El objetivo del trabajo fue evaluar las características agronómicas y rendimiento de cinco variedades de poroto en dos localidades del Chaco Central del Paraguay, de tal forma a identificar las más productivas. La evaluación se realizó entre los meses de enero y abril de 2013. Los tratamientos estuvieron constituidos por la combinación de dos localidades (Isla Poi y Betania) y cinco variedades de poroto (San Francisco'i, Pyta'i, San Francisco Guazú, Blanco Ojo Negro y Moteado) totalizando 10 tratamientos. El diseño experimental fue de bloques completos al azar con arreglo factorial 2 x 5 y cuatro repeticiones. Cada unidad experimental estuvo constituida por cuatro hileras de $4 \mathrm{~m}$ de longitud, separadas entre sí 0,70 m. Luego del raleo se dejaron dos plantas por hoyo cada $0,25 \mathrm{~m}$. Las variables evaluadas fueron rendimiento de granos con vainas, rendimiento de granos secos, porcentaje de rendimiento de granos secos, número de granos por vaina y peso de 100 semillas. Los análisis estadísticos muestran que en Isla Poi las variedades más productivas fueron Pyta'i $\left(4,40 \mathrm{t} \mathrm{ha}^{-1}\right)$, San Francisco Guazú $\left(4,27 \mathrm{t} \mathrm{ha}^{-1}\right)$ y San Francisco'i $\left(3,81 \mathrm{t} \mathrm{ha}^{-1}\right)$, mientras que en Betania todas las variedades presentaron rendimiento estadísticamente similares que oscilaron entre $1,19 \mathrm{t} \mathrm{ha}^{-1}$ (San Francisco'i) y 1,53 t ha ${ }^{-1}$ (Pyta'i). En la localidad de Isla Poi los rendimientos fueron superiores. Las variedades Moteado y Blanco Ojo Negro presentaron rendimientos más estables en las dos localidades.

Palabras clave: Vigna unguiculata (L.) Walp, rendimiento, variedades.

\begin{abstract}
Cowpea are one of the most consumed legumes in Paraguay, produced for both on-farm consumption as well as an important cash-crop for family farming. The aim of this work was to evaluate agronomical characteristics and yield of five varieties of cowpea in 2 locations of the Paraguayan Central Chaco, in order to identify those with the highest yields. The evaluation was carried out between January and April, 2013. The trials were conducted by combining two locations (Isla Po'i and Betania) and five varieties of cowpea (San Francisco'i, Pyta'i, San Francisco Guazú, Blanco Ojo Negro y Moteado) totaling 10 trials. The experimental design was randomized complete blocks, 2 x 5 factorial arrangements with four repetitions. Each experimental unit was made up of four 4 meters long rows, separated by 0,7 meters. After thinning, two plants per planting hole were left standing every $0,25 \mathrm{~m}$. The variables evaluated were grain yield per bean pod, yield of dry grain, percentage of dry grain, number of grain per pod, and the weight of 100 seeds. The statistical analysis shows that in Isla Po'i the more productive varieties were Pyta'i $\left(4,40 \mathrm{t} \mathrm{ha}^{-1}\right)$, San Francisco Guazú $\left(4,27 \mathrm{t} \mathrm{ha}^{-1}\right)$ and San Francisco'i (3,81 t $\left.\mathrm{ha}^{-1}\right)$, while in Betania all varieties showed statistically similar results that varied between $1,19 \mathrm{t} \mathrm{ha}^{-1}$ (San Francisco'i) and 1,53 $\mathrm{t} \mathrm{ha}^{-1}$ (Pyta'i). In Isla Po'i yields were higher. The Moteado and Blanco Ojo Negro varieties are more stable in their yields in the two locations evaluated, with no effect on their production. Key words: Vigna unguiculata (L.) Walp, variety, yield.
\end{abstract}




\section{INTRODUCCIÓN}

El poroto Vigna unguiculata (L.) Walp., también es conocido localmente como "kumanda" en guaraní o "caupi" en español y portugués. Esta última denominación, deriva de la palabra inglesa cowpea, es una leguminosa nativa de regiones áridas (Singh 2005), que en la actualidad se encuentra difundida en todas las áreas tropicales y subtropicales.

Es un cultivo multifuncional, ya que es una fuente de alimentos para el hombre, además es utilizado en la ganadería como forraje y sirve como una valiosa y confiable mercancía de generación de ingresos para los agricultores (Timko y Singh 2008, Langyintuo et al. 2003). Tradicionalmente considerado como rubro de autoconsumo y de renta en la agricultura familiar paraguaya. $\mathrm{Su}$ cultivo es realizado en todos los departamentos del país, y el MAG/DCEA (2013), en el año agrícola 2012/13 estimó una superficie de siembra de $70.000 \mathrm{ha}$, con rendimiento promedio de $0,8 \mathrm{t} \mathrm{ha}^{-1}$.

Los granos del poroto son muy apreciados por el elevado contenido de proteínas, razón por lo cual constituye una fuente de alimentos muy importante para el consumo humano, ya sea en forma de grano fresco, seco o harina (López Guerra y Bressani 2008). La utilización de proteínas de origen vegetal para consumo humano se ha difundido en los países en vías de desarrollo, debido al elevado costo de las proteínas de origen animal (Iqbal et al. 2006, Miquilena e Higuera Moros 2012). Además de su elevado contenido en proteínas (23 a 25\%), posee carbohidratos, alto tenor de fibras alimentarias, vitaminas, minerales y un bajo porcentaje de lípidos (2\%) (EMBRAPA 2003), así también la planta fija una importante cantidad de nitrógeno en el suelo (Asiwe 2009).

Los principales nutrientes extraídos por el poroto en orden de importancia son el Potasio, Nitrógeno, Calcio, Magnesio y Fosforo, pero pueden presentarse varios padrones de respuesta por la variabilidad genética existente en el cultivo (Oliveira 1988). En regiones donde la fertilidad del suelo es baja, la precipitación es limitante y la mayor parte del cultivo de poroto se realiza sin el uso de fertilizantes y medidas fitosanitarias (pesticidas o herbicidas), una amplia variedad de factores bióticos y abióticos pueden limitar su crecimiento y reducir severamente el rendimiento (Singh 2005, Timko y Singh 2008).
El Chaco Central del Paraguay se caracteriza por presentar una precipitación media anual de $850 \mathrm{~mm}$, evaporación que puede llegar hasta $1.699 \mathrm{~mm} \mathrm{año}^{-1}$, lo cual indica la existencia de periodos con déficit hídrico y temperatura media de $25^{\circ} \mathrm{C}$ (Harder et al. 2004, Glatzle 1999).

En esa región, el poroto, puede constituirse en una alternativa para el cultivo, por sus cualidades nutricionales, rusticidad, excelente adaptación a suelos arenosos, tolerante a las condiciones de altas temperaturas y sequías, propias de los trópicos (Ehlers y Hall 1997; Vural y Karusu 2007, Silva et al. 2012). Además, se constituye en un rubro estratégico para la seguridad alimentaria de las poblaciones donde existen familias de escasos recursos, cuya alimentación es baja en nutrientes, requiriendo de alimentos ricos en proteínas en sus dietas para cubrir sus necesidades básicas de nutrición.

Debido a la importancia de este rubro y la escasa información científica sobre el comportamiento de variedades de poroto en las condiciones climáticas de esa zona, se realizó esta investigación con el objetivo de evaluar las características agronómicas y rendimiento de cinco variedades de poroto en dos localidades del Chaco Central, de tal forma a identificar las más productivas.

\section{MATERIALES Y MÉTODOS}

La investigación se realizó en dos localidades del Chaco Central del Paraguay. La primera, corresponde a la Chacra Experimental Isla Poi, del Servicio Agropecuario de la Sociedad Cooperativa Chortitzer Ltda., situado en el Departamento de Boquerón, distrito de Loma Plata, cuyas coordenadas geográficas son $22^{\circ} 29^{\prime} 38,7^{\prime}$ 'latitud sur y $59^{\circ} 43^{\prime} 53,2^{\prime}$ 'longitud oeste. La segunda localidad, fue la finca de un productor de la aldea Betania, comunidad "Yalve Sanga", distrito de Loma Plata, Departamento de Boquerón, cuyas coordenadas son: $22^{\circ} 36^{\prime} 10,2^{\prime}$ ' latitud sur y $59^{\circ} 48^{\prime} 38,0^{\prime}$ ' longitud oeste.

Las medias semanales de temperatura y precipitación, recabadas en la Estación Meteorológica de la Chacra Experimental Isla Poi, desde la siembra el 4 de enero, hasta la última cosecha el 25 de abril de 2013 se presentan en la figura 1, mientras que los datos de Betania, desde la siembra el 15 de febrero de 2013 hasta la cosecha el 29 de abril de 2013 se presentan en la figura 2. En la localidad de Isla Poi, en el ciclo agrícola anterior al experimento, fue sembrado soja y posterior a la cosecha se realizó el barbecho químico para facilitar la acumulación de agua en 
los perfiles inferiores. El barbecho químico consistió en mantener la superficie del terreno libre de malezas, mediante la aplicación de herbicidas de acción total, de manera a evitar el consumo de agua por las malezas y se encuentre disponible para el siguiente cultivo a ser implantado. En Betania, la parcela donde se instaló el experimento estaba en descanso por tres años y enmalezado con Poaceas.
Las muestras de suelo extraídas de ambas localidades, fueron analizadas en el Laboratorio de Suelos de la Facultad de Ciencias Agrarias (FCA) de la Universidad Nacional de Asunción (UNA), cuyos resultados se presentan en la tabla 1 .

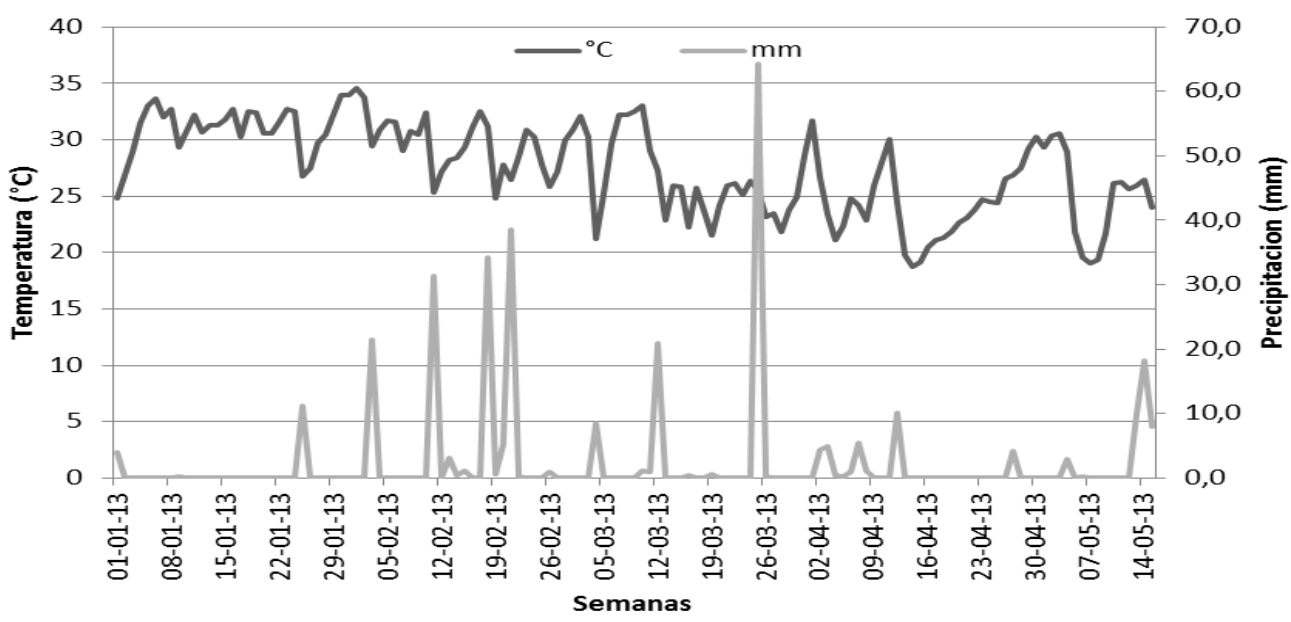

Figura 1. Temperatura y precipitación media semanal desde la siembra hasta la cosecha de variedades de poroto en Isla Poi. 2013.

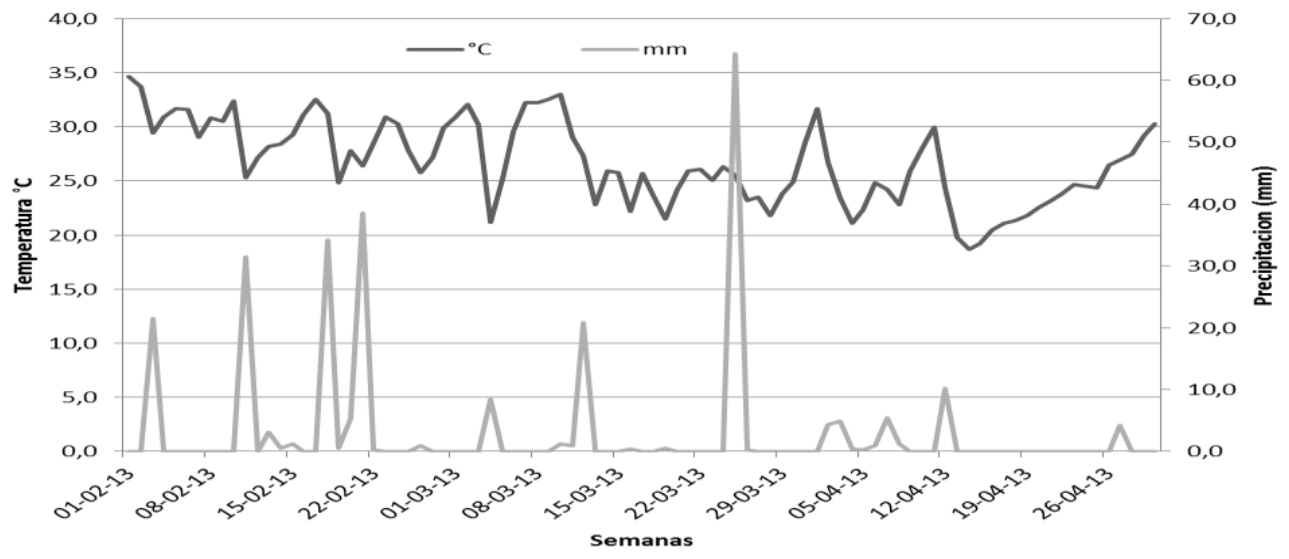

Figura 2. Temperatura y precipitación media semanal desde la siembra hasta la cosecha de variedades de poroto en Betania. 2013.

Tabla 1. Resultados del análisis de suelo de las parcelas experimentales de Isla Poi y Betania. 2013.

\begin{tabular}{|c|c|c|c|c|c|c|c|c|}
\hline J & pH & Materia orgánica & $\mathbf{P}$ & $\mathrm{Ca}^{+2}$ & $\mathrm{Mg}^{+2}$ & $\mathbf{K}^{+}$ & $\mathrm{Al}^{+3}+\mathrm{H}^{+}$ & Textura \\
\hline Local & & $(\%)$ & ppm & $\mathrm{cmol}_{\mathrm{c}} / \mathrm{kg}$ & $\mathrm{cmol}_{\mathrm{c}} / \mathrm{kg}$ & $\mathrm{cmol}_{\mathrm{c}} / \mathrm{kg}$ & $\mathrm{cmol}_{\mathrm{c}} / \mathrm{kg}$ & \\
\hline Isla Poi & 7,35 & 2,80 & 62,96 & 2,33 & 0,96 & 0,50 & 0,0 & Arenosa \\
\hline Betania & 6,34 & 2,65 & 4,20 & 0,78 & 0,46 & 0,19 & 0,0 & Arenosa \\
\hline
\end{tabular}


Los tratamientos estuvieron constituidos por la combinación de las localidades donde se realizó la investigación (Isla Poi y Betania) y las cinco variedades de poroto (San Francisco'i; Pyta'i; San Francisco Guazú; Blanco Ojo Negro y Moteado). El diseño experimental fue de bloques completos, dispuesto en un arreglo factorial 2 × 5, con cuatro repeticiones. Cada unidad experimental estuvo constituida por cuatro hileras de $4 \mathrm{~m}$ de longitud.

En ambas localidades la preparación de suelo se efectuó en forma convencional mediante arada y rastreada. Antes de la siembra se procedió a abrir surcos de aproximadamente $5 \mathrm{~cm}$ de profundidad, separados entre sí cada $0,70 \mathrm{~m}$, donde fueron depositadas tres semillas cada 0,25 m y luego de dos semanas de la emergencia, se efectuó el raleo dejando dos plantas por hoyo.

Durante el desarrollo del cultivo se realizaron dos carpidas en forma manual y se aplicó Cipermetrina en dos oportunidades, utilizando dosis de $1 \mathrm{~cm}^{3} \mathrm{~L}^{-1}$ de agua, para el control de pulgón (Aphis spp). Las parcelas experimentales no fueron irrigadas ni fertilizadas.

La cosecha de las diferentes variedades en ambas localidades se realizó en forma manual, cuando las vainas estaban totalmente secas. En la localidad de Isla Poi, la primera cosecha se realizó a los 77 días después de la siembra, la segunda 97 días después y la última a los 111 días, totalizando tres cosechas, mientras que en Betania, todas las variedades fueron cosechadas en una sola oportunidad, a los 74 días después de la siembra.

Fueron cosechadas las dos hileras centrales de cada unidad experimental, e inmediatamente se procedió al pesaje de las vainas con granos (rendimiento de granos con vainas) $y$, posteriormente fueron llevadas a una pista de secado, en la Chacra Experimental Isla Poi donde permanecieron por 48 horas, y finalmente se procedió al desgrane en forma manual.

Las variedades fueron evaluadas en función a las siguientes características: rendimiento de granos con vainas, porcentaje de rendimiento de granos (cociente entre rendimiento de granos secos y el rendimiento de granos con vainas, multiplicado por 100), rendimiento de granos secos, número de granos por vaina y peso de 100 semillas (extraídas al azar de los granos secos). Para la evaluación del número de granos se utilizaron 10 vainas por tratamiento y repetición.
Los datos fueron analizados estadísticamente mediante el análisis de varianza y, cuando los factores presentaron efectos significativos $(p<0,05)$, las medias fueron comparadas por la prueba de Tukey al 5\% de probabilidad. El paquete estadístico utilizado fue el MSTAT.

\section{RESULTADOS Y DISCUSIÓN}

El rendimiento de granos con vainas presentó diferencias estadísticas significativas para variedades, localidades y la interacción entre factores. Las variedades San Francisco'i, Pyta'i y San Francisco Guazú generaron mayor rendimiento de granos con vainas en la localidad de Isla Poi, comparados al rendimiento obtenido en Betania, mientras que Blanco Ojo Negro y Moteado no difirieron en su rendimiento en las dos localidades, lo cual podría indicar que no fueron afectados por el ambiente. Analizando el comportamiento de las variedades en Isla Poi, se constató que Pyta'i $\left(6,50 \mathrm{t} \mathrm{ha}^{-1}\right)$, San Francisco Guazú $\left(6,12 \mathrm{t} \mathrm{ha}^{-1}\right)$ y San Francisco'i (5,96 $\left.\mathrm{t} \mathrm{ha}^{-1}\right)$, presentaron las mayores medias y estadísticamente fueron superiores a Blanco Ojo Negro $\left(2,55 \mathrm{t} \mathrm{ha}^{-1}\right)$ y Moteado $\left(3,43 \mathrm{t} \mathrm{ha}^{-1}\right)$. Las variedades Moteado y Blanco Ojo Negro, a su vez no fueron diferentes entre sí estadísticamente (Tabla 2).

En la localidad de Betania no hubo diferencias estadísticas significativas entre variedades. Las medias registradas fueron de 2,23 $\mathrm{t} \mathrm{ha}^{-1}$ (Blanco Ojo Negro), 2,21 t ha ${ }^{-1}$ (Pyta'i), 2,12 $\mathrm{t} \mathrm{ha}^{-1}$ (Moteado), 1,93 t ha ${ }^{-1}$ (San Francisco Guazú) y 1,81 t ha ${ }^{-1}$ (San Francisco'i). Por otro lado, al comparar las medias de las variedades en las dos localidades se encontró que Pyta'i (4,35 t ha ${ }^{-}$ $\left.{ }^{1}\right)$, San Francisco Guazú $\left(4,02 \mathrm{t} \mathrm{ha}^{-1}\right)$ y San Francisco'i $\left(3,88 \mathrm{t} \mathrm{ha}^{-1}\right)$ fueron las más productivas $\mathrm{y}$ estadísticamente superiores a Blanco Ojo Negro (2,38 t $\mathrm{ha}^{-1}$ ) y Moteado $\left(2,80 \mathrm{t} \mathrm{ha}^{-1}\right)$. Considerando las medias de las variedades en las localidades donde se realizó la investigación, se observó que en Isla Poi la media fue de 4,91 $\mathrm{t} \mathrm{ha}^{-1}$, superior estadísticamente a la obtenida en Betania que fue de 2,07 $\mathrm{t} \mathrm{ha}^{-1}$ (Tabla 2).

La productividad de granos con vainas estuvo entre 6,50 y 2,55 $\mathrm{t} \mathrm{ha}^{-1}$ en Isla Poi y 2,23 y $1,81 \mathrm{t} \mathrm{ha}^{-1}$ en Betania. Oliveira et al. (2002), encontraron en el municipio de Areias, estado de Paraíba, Brasil que la línea de poroto CNCX-409-127 fue la más productiva con rendimiento de $6,5 \mathrm{t} \mathrm{ha}^{-1}$ de granos con vainas, coincidiendo con el rendimiento de la variedad más productiva en Isla Poi, que fue Pyta'i. 
Tabla 2. Rendimiento de granos con vainas $\left(\mathrm{t} \mathrm{ha}^{-1}\right)$ de cinco variedades de poroto evaluadas en dos localidades del Chaco Central. 2013.

\begin{tabular}{|c|c|c|c|}
\hline Variedad/Localidad & Isla Poi & Betania & Media Variedad \\
\hline San Francisco’i & $5,96 \mathrm{Aa}$ & $1,81 \mathrm{Ab}$ & $3,88 \mathrm{~A}$ \\
\hline Pyta'i & $6,50 \mathrm{Aa}$ & $2,21 \mathrm{Ab}$ & $4,35 \mathrm{~A}$ \\
\hline San Francisco Guazú & $6,12 \mathrm{Aa}$ & $1,93 \mathrm{Ab}$ & $4,02 \mathrm{~A}$ \\
\hline Blanco Ojo Negro & $2,55 \mathrm{Ba}$ & $2,23 \mathrm{Aa}$ & $2,38 \mathrm{~B}$ \\
\hline Moteado & $3,43 \mathrm{Ba}$ & $2,12 \mathrm{Aa}$ & $2,80 \mathrm{~B}$ \\
\hline Media Localidad & $4,91 \mathrm{a}$ & $2,07 \mathrm{~b}$ & \\
\hline F. cal.: Localidad $322,3^{* *}$ & \multicolumn{3}{|c|}{ CV(\%): Localidad 14,31 } \\
\hline F. cal.: Variedad $20,4^{* *}$ & \multicolumn{3}{|c|}{$\mathrm{CV}(\%)$ : Variedad 15,22} \\
\hline F. cal.: Variedad x localidad $18,79^{* *}$ & \multicolumn{3}{|c|}{$\mathrm{CV}(\%)$ : Variedad x localidad 17,71} \\
\hline
\end{tabular}

**Significativo al 1\% de probabilidad de error por la prueba de F. Letras minúsculas iguales en las filas y mayúsculas en las columnas no difieren entre sí por la prueba de Tukey al 5\% de probabilidad de error.

Para rendimiento de granos secos se encontró interacción significativa entre variedades y localidades. Las variedades San Francisco'i, Pyta'i y San Francisco Guazú con medias de 3,81 $\mathrm{t} \mathrm{ha}^{-1}, 4,40 \mathrm{t} \mathrm{ha}^{-1}$ y 4,27 $\mathrm{t} \mathrm{ha}^{-1}$, respectivamente, fueron más productivas en la localidad de Isla Poi y difirieron del rendimiento en Betania, donde las mismas variedades produjeron $1,19 \mathrm{t} \mathrm{ha}^{-1}, 1,53 \mathrm{t} \mathrm{ha}^{-1} \mathrm{y}$ $1,37 \mathrm{t} \mathrm{ha}^{-1}$, respectivamente. Por otro lado, el rendimiento de granos secos obtenidos con las variedades Blanco Ojo Negro $\left(1,72 \mathrm{t} \mathrm{ha}^{-1}\right)$ y Moteado $\left(2,33 \mathrm{t} \mathrm{ha}^{-1}\right)$ en la localidad de Isla Poi no fueron estadísticamente diferentes del rendimiento en Betania, donde las mismas variedades produjeron $1,49 \mathrm{t} \mathrm{ha}^{-1}$ y $1,47 \mathrm{t} \mathrm{ha}^{-1}$, lo cual indica que el rendimiento de dichas variedades no fue influenciado por los ambientes donde se desarrolló la investigación (Tabla 3).

El rendimiento de granos de las variedades en Isla Poi, presentó diferencias estadísticas significativas, destacándose la variedad Pyta'i con una productividad de 4,40 $\mathrm{t} \mathrm{ha}^{-1}$, superando a Moteado (2,33 $\left.\mathrm{t} \mathrm{ha}^{-1}\right)$ y Blanco Ojo Negro $\left(1,72 \mathrm{t} \mathrm{ha}^{-1}\right)$, pero estadísticamente similar a San Francisco Guazú (4,27 t ha $\left.{ }^{-1}\right)$ y San Francisco'i $(3,81$ $\mathrm{t} \mathrm{ha}^{-1}$ ) indicando que esas variedades fueron más eficientes en la distribución de granos dentro de las vainas y también en el aprovechamiento de los foto asimilados (Addo-Quaye 2011).

Tabla 3. Rendimiento de granos secos $\left(\mathrm{t} \mathrm{ha}^{-1}\right)$ de cinco variedades de poroto evaluadas en dos localidades del Chaco Central. 2013.

\begin{tabular}{lccc}
\hline Variedad & Isla Poi & Betania & Media Variedad \\
\hline San Francisco'i & $3,81 \mathrm{Aa}$ & $1,19 \mathrm{Ab}$ & $2,50 \mathrm{~A}$ \\
Pyta'i & $4,40 \mathrm{Aa}$ & $1,53 \mathrm{Ab}$ & $2,97 \mathrm{~A}$ \\
San Francisco Guazú & $4,27 \mathrm{Aa}$ & $1,37 \mathrm{Ab}$ & $2,83 \mathrm{~A}$ \\
Blanco Ojo Negro & $1,72 \mathrm{Ba}$ & $1,49 \mathrm{Aa}$ & $1,60 \mathrm{~B}$ \\
Moteado & $2,33 \mathrm{Ba}$ & $1,47 \mathrm{Aa}$ & $1,90 \mathrm{~B}$ \\
\hline Media localidad & $3,30 \mathrm{a}$ & $1,41 \mathrm{~b}$ & \\
\hline F. cal.: Localidad 206,3** & $\mathrm{CV}(\%)$ : Localidad 17,67 \\
F. cal.: Variedad 18,9* & $\mathrm{CV}(\%)$ : Variedad 16,26 \\
F.cal.: Variedad x localidad 14,5* & $\mathrm{CV}(\%)$ : Variedad x localidad 19,74
\end{tabular}

*Significativo al 5\% de probabilidad de error por la prueba de F. **Significativo al $1 \%$ de probabilidad de error por la prueba de F. Letras minúsculas iguales en las filas y mayúsculas en las columnas no difieren entre sí por la prueba de Tukey al 5\% de probabilidad de error. 
En la localidad Betania, no existieron diferencias estadísticas en el rendimiento de granos secos entre las variedades, encontrándose medias de $1,19 \mathrm{t} \mathrm{ha}^{-1}$ (San Francisco'i), 1,53 t ha ${ }^{-1}$ (Pyta'i), 1,37 t ha ${ }^{-1}$ (San Francisco Guazú), 1,49 t ha ${ }^{-1}$ (Blanco Ojo Negro) y 1,47 $\mathrm{t} \mathrm{ha}^{-1}$ (Moteado).

Comparando el comportamiento en términos de la producción de granos secos de las variedades entre las dos localidades (Tabla 3), se encontró mayor productividad en Isla Poi, donde la media general del experimento fue de 3,30 $\mathrm{t} \mathrm{ha}^{-1}$, difiriendo estadísticamente de la media obtenida en Betania $\left(1,41 \mathrm{t} \mathrm{ha}^{-1}\right)$. La productividad en ambas localidades es muy superior a la media nacional de 0,8 $\mathrm{t} \mathrm{ha}^{-1}$ (MAG/DCEA 2013), lo cual evidencia el potencial de la región para el cultivo del poroto.

El mayor rendimiento en Isla Poi, puede atribuirse al manejo diferenciado de suelo realizado en la misma, donde la parcela experimental estuvo con barbecho químico, lo cual permitió la acumulación de agua en capas más profundas del suelo y de esa forma posibilitó el suministro constante al cultivo para cubrir sus necesidades y así expresar su potencial productivo. En estudios realizados en el Chaco Central por Rabery y Dyck (2011) encontraron que el barbecho químico aumenta la cantidad de agua acumulada en el suelo, permitiendo que las raíces de las plantas, tengan mayor crecimiento y exploren mayores profundidades del suelo. Los resultados demuestran que el empleo del barbecho químico es una práctica que permite acumular agua en el suelo y contribuye para que el cultivo principal crezca y se desarrolle mejor en las condiciones del Chaco Central.

Además, en Isla Poi, la acumulación de agua en el suelo, permitió el aumento de la cantidad de cosechas, realizándose un total de tres, el ciclo del cultivo fue de 111 días, mientras que en Betania, se efectuó una sola cosecha y el ciclo total fue de 74 días. En la localidad de Isla Poi durante el ciclo del cultivo se tuvo precipitación de $278 \mathrm{~mm}$, con una mejor distribución durante el periodo de crecimiento vegetativo, floración y formación de vainas (Figura 1), lo cual favoreció el aumento en el número de cosechas y como consecuencia el rendimiento. En el experimento conducido en Betania en el periodo comprendido entre la siembra y cosecha se tuvo una precipitación de 209,9 mm, lo que representa $68,0 \mathrm{~mm}$ menos que en Isla Poi (Figura 2).

En Isla Poi, sumado al barbecho químico, la parcela utilizada presentó un suelo con niveles más elevados en nutrientes como fósforo, potasio, calcio y magnesio, probablemente influenciado por el cultivo anterior con soja, que recibió fertilización química (Tabla 1). En ese sentido, Brito Melo et al. (2005) señalan que el fósforo a pesar de ser extraído en menor cantidad por el poroto, comparado con otros macronutrientes, es el limitante en la producción del cultivo. Esta limitación en fosforo ejerce un efecto importante sobre las leguminosas de granos que dependen de la fijación simbiótica del $\mathrm{N}_{2}$ para su crecimiento, debido a que cuando se nutren de nitrógeno por esta vía requieren dosis más altas de fertilizantes fosfóricos que cuando reciben $\mathrm{N}$ en forma mineral (Israel 1987). Además, en diversas investigaciones se encontró respuestas positivas en términos de rendimiento a su aplicación (Alves et al. 2001, Silva et al. 2010).

La existencia de diferencias estadísticas en la producción de granos secos entre las variedades de poroto evaluadas, concuerda con la investigación realizada en el estado de Paraíba, Brasil por Santos et al. (2009). Sin embargo, dichos autores obtuvieron con la variedad más productiva un rendimiento de $1,23 \mathrm{t} \mathrm{ha}^{-1}$, que es inferior a todas las medias registradas en esta investigación, exceptuando el rendimiento en Betania con la variedad San Francisco'i $\left(1,19 \mathrm{tha}^{-1}\right)$.

El rendimiento de granos secos obtenido con las cinco variedades de poroto en Isla Poi estuvieron entre 4,40 t $\mathrm{ha}^{-1}$ y $1,71 \mathrm{t} \mathrm{ha}^{-1} \mathrm{y}$ en Betania entre $1,53 \mathrm{t} \mathrm{ha}^{-1}$ y 1,19 $\mathrm{t} \mathrm{ha}^{-1}$ (Tabla 3). En investigaciones realizadas en Mossoró, Brasil, por Silva y Montenegro (1997) con cuatro genotipos de poroto que recibieron fertilización química con NPK, no encontraron diferencias estadísticas entre variedades, y reportaron rendimientos entre $1,08 \mathrm{t} \mathrm{ha}^{-1} \mathrm{y}$ $1,43 \mathrm{t} \mathrm{ha}^{-1}$. Oliveira et al. (2002) comparando 13 genotipos de poroto en el Brasil, encontraron diferencias entre materiales y las medias oscilaron en un rango de 0,60 a $1,80 \mathrm{tha}^{-1}$.

El porcentaje del rendimiento de granos secos, respecto al peso de granos con vainas de las variedades de poroto evaluadas en este trabajo, solamente presentó diferencias significativas entre variedades por la prueba de $\mathrm{F}$ al $5 \%$ de probabilidad de error (Tabla 4). Sin embargo, aplicando la prueba de Tukey al 5\% de probabilidad no se encontraron diferencias significativas. Las medias estuvieron entre 64,63\% (San Francisco'i) y 70,25\% (San Francisco Guazú). Estos valores son inferiores a la media de $75 \%$ de rendimiento en granos que presenta el poroto (Jover 2003). 
Tabla 4. Porcentaje de rendimiento de granos, de cinco variedades de poroto evaluadas en dos localidades del Chaco Central. 2013.

\begin{tabular}{|c|c|}
\hline Variedad & Medias (\%) \\
\hline San Francisco'i & 64,63 \\
\hline Pyta'i & 68,63 \\
\hline San Francisco Guazú & 70,25 \\
\hline Blanco Ojo Negro & 67,25 \\
\hline Moteado & 68,05 \\
\hline \multicolumn{2}{|l|}{ Localidad } \\
\hline Isla Poi & 67,30 \\
\hline Betania & 68,05 \\
\hline F. cal.: Localidad $0,35^{\text {ns }}$ & CV(\%): Localidad 5,90 \\
\hline F. cal.: Variedad $7,82^{*}$ & CV $(\%)$ : Variedad 3,08 \\
\hline F.cal.: Variedad x localidad $0,41^{\text {ns }}$ & CV $(\%)$ : Variedad x localidad 3,44 \\
\hline
\end{tabular}

El peso de cien semillas, de las variedades de poroto estudiadas, presentó interacción significativa entre localidades y variedades (Tabla 5). San Francisco'i, Pyta'i y San Francisco Guazú generaron las menores medias en
Isla Poi, mientras que la variedad Blanco Ojo Negro en Betania. Moteado presentó media de 12,40 g en Isla Poi y $14,05 \mathrm{~g}$ en Betania, sin que existan diferencias significativas entre las localidades.

Al analizar el comportamiento de las variedades para esta variable, en Isla Poi se verificó que San Francisco Guazú $(19,60$ g) generó la mayor media y difirió de las demás variedades. Pyta'i con 15,5 g fue superior a Blanco Ojo Negro (12,90 g), Moteado (12,40 g) y San Francisco’i $(11,0 \mathrm{~g})$. Esta última presentó la menor media y estadísticamente fue similar a Moteado. En la localidad de Betania, San Francisco Guazú (20,50 g) fue superior a las demás, en segundo lugar se posicionó la variedad Pyta'i $(16,15 \mathrm{~g})$, que difirió estadísticamente de San Francisco'i $(12,35 \mathrm{~g})$, Moteado (14,05 g) y Blanco Ojo Negro (10,60 g). Considerando las medias de las variedades en las localidades, San Francisco Guazú (20,05 g) ocupó el primer lugar, difiriendo estadísticamente de las otras variedades, seguido de Pyta'i y Moteado. Blanco Ojo Negro y San Francisco'i generaron las menores medias y estadísticamente son similares entre sí. Entre las localidades, no se detectaron diferencias significativas (Tabla 5).

Tabla 5. Peso de cien semillas (gramos) de cinco variedades de poroto en dos localidades del Chaco Central. 2013.

\begin{tabular}{|c|c|c|c|}
\hline Variedad & Isla Poi & Betania & Media Variedad \\
\hline San Francisco’i & $11,00 \mathrm{D} \mathrm{b}$ & $12,35 \mathrm{CD}$ a & $11,68 \mathrm{D}$ \\
\hline Pyta'i & $15,55 \mathrm{~B} \mathrm{~b}$ & $16,15 \mathrm{~B}$ a & $15,85 \mathrm{~B}$ \\
\hline San Francisco Guazú & $19,60 \mathrm{~A} \mathrm{~b}$ & $20,50 \mathrm{~A}$ a & $20,05 \mathrm{~A}$ \\
\hline Blanco Ojo Negro & $12,90 \mathrm{C} \mathrm{a}$ & $10,60 \mathrm{D} \mathrm{b}$ & $11,75 \mathrm{D}$ \\
\hline Moteado & $12,40 \mathrm{CD}$ a & $14,05 \mathrm{C} \mathrm{a}$ & $13,23 \mathrm{C}$ \\
\hline Media localidad & $14,29 \mathrm{a}$ & $14,73 \mathrm{a}$ & \\
\hline F. cal.: Localidad $6,78^{\text {ns }}$ & & \multicolumn{2}{|c|}{ CV(\%): Localidad 3,68 } \\
\hline F. cal.: Variedad $304,39 * *$ & & \multicolumn{2}{|c|}{$\mathrm{CV}(\%):$ Variedad 3,94 } \\
\hline F.cal.: Variedad $\mathrm{x}$ localidad $8,48^{*}$ & & \multicolumn{2}{|c|}{ CV(\%): Variedad x localidad 5,30 } \\
\hline
\end{tabular}

Estos resultados son coincidentes con los obtenidos en Mossoró, Brasil, por Torres et al. (2008) que al comparar 10 accesiones de poroto encontraron medias que oscilaron entre 15,86 y 23,47 g. Por su parte Santos et al. (2009), evaluando cuatro variedades de poroto, reportaron valores entre $20,11 \mathrm{~g}$ y $32,86 \mathrm{~g}$ por cada cien semillas. Gutiérrez et al. (2001) comparando el peso de cien semillas de poroto en diferentes sistemas de manejo de suelo encontraron medias entre 21,95 y 20,21 g. En esta investigación en la localidad de Isla Poi, las medias variaron de 19,60 g (San Francisco Guazú) a 11,00 g (San Francisco’i), mientras que en Betania de 20,50 g (San Francisco Guazú) a 10,60 g (Blanco Ojo Negro), inferiores a las investigaciones citadas 
precedentemente. Freire Filho et al. (1981) verificaron que el intervalo medio para esta variable es de $19,46 \pm 3,89 \mathrm{~g}$.

El peso es considerado como una medida del tamaño de la semilla y se ha encontrado que es altamente heredable (Fery 1985). Algunos autores atribuyen el peso de las semillas al contenido de materia orgánica del suelo, independiente de la fertilización química, por el mejoramiento de otros componentes de la fertilidad del suelo, como disponibilidad de agua, aumento de capacidad de intercambio catiónico (Marchesini et al. 1998, Yamada y Kamata 1989, Kamara 1976).

El número de granos por vaina no presentó diferencias estadísticas significativas entre variedades, localidades y en la interacción de factores. Las medias de las variedades evaluadas estuvieron entre 13,62 y 15,5 granos por vaina, que se encuentran comprendidas en el rango de 12 a 16 semillas por vaina, citado por Torres et al. (2008) al comparar 10 accesiones de poroto. Santos et al. (2009) evaluando cuatro variedades de poroto reportaron diferencias estadísticas para dicha variable, con medias entre 7,56 y 16,40 granos por vaina.

Addo-Quaye et al. (2011) evaluando variedades de poroto concluyeron que diferentes localidades pueden no afectar el número de granos por vaina, lo cual implica que esta característica también es controlada genéticamente por la variedad y que las condiciones ambientales pueden tener o no influencia sobre la misma.

\section{CONCLUSIONES}

En la localidad de Isla Poi, las variedades mejor adaptadas son Pyta'i, San Francisco Guazú y San Francisco'i, mientras que en Betania cualquiera de las variedades evaluadas pueden ser cultivadas, ya que presentaron medias similares. Las variedades Moteado y Blanco Ojo Negro son las más estables en su rendimiento.

Todas las variedades presentan similar número de granos por vaina. Así también, el cultivo en localidades diferentes no afecta el rendimiento de granos expresado en porcentaje, el número de granos por vaina y el peso de 100 semillas.

\section{AGRADECIMIENTOS}

Los autores agradecen al Consejo Nacional de Ciencia y Tecnología (CONACYT) por financiar este trabajo de investigación.

\section{REFERENCIAS BIBLIOGRÁFICAS}

Addo-Quaye, AA; Darkwa, AA; Ampiah, MKP. 2011. Performance of three cowpea (Vigna unguiculata (L) Walp) varieties in two agro-ecological zones of the central region of Ghana: grain yield and its components. Journal of Agricultural and Biological Science 6 (2):34-42.

Alves, JMA; Albuquerque, J de AA; Uchôa, SCP; Silva, AJ da; Silva, LC da; Santos, EG dos. 2001. Componentes de produção de uma linhagem de feijão-caupi precoce consorciada com a mandioca no Lavrado de Roraima In: V Reunião Nacional de Pesquisa de Caupi. Teresina-PI. p. $98-101$.

Asiwe, JAN. 2009. Needs Assessment of Cowpea Production Practices, Constraints and Utilization in South Africa. African Journal of Biotechnology 8 (20):5383- 5388 .

Brito Melho, F; Cardoso, MJ; Salviano, AAC. 2005. Fertilidade do solo. In: Feijão-caupi: avanços tecnológicos. Freire Filho, FR; Lima, JAA; Riveiro, VQ. Eds. Brasília: Embrapa Informação Tecnológica. BR. p. 231-42.

Ehlers, JD; Hall, AE. 1997. Caupí [Vigna unguiculata (L.) Walp.]. Field Crops Res. 53:87-204.

EMBRAPA (Empresa Brasileira de Pesquisa Agropecuaria). 2003. Cultivo de feijão caupi. Consultado 17 may 2015. Disponible en: http://sistemasdeproducao.cnptia.embra pa.br/FontesHTML/Feijao/FeijaoCaupi/

Fery, RL. 1985. The genetics of cowpea. A review of the world literature. In: Singh, SR; Rachie, KO (eds). Cowpea research, production and utilization. Wiley, Chichester, UK. p. 25-62.

Freire Filho, FR; Cardoso, MJ; Araújo, AG de; Santos, AA dos, Silva, PHS da. 1981. Características botânicas e agronômicas de cultivares de feijão macássar (Vigna unguiculata (L.) Walp.). Teresina: EMBRAPA-UEPAE de Teresina. 45p. (EMBRAPA - UEPAE de Teresina. Boletim de Pesquisa, 4).

Glatzle, A. 1999. Compendio para el manejo de pasturas en el Chaco. Chaco Central, PY: El Lector. 188. p.

Gutiérrez, W; Medrano, C; Materan, M; Villalobos, Y; Esparza, D; Báez, J; Medina, B. 2001. Evaluación del rendimiento y nodulación del frijol Vigna unguiculata (L.) Walp bajo dos sistemas de labranza en las 
condiciones agroecológicas de la planicie de Maracaibo. Rev Fac Agron (LUZ) 18: 237-246.

Harder, W; Thiessen, H; Klassen, N. 2004. Libreto de agua: colecta, almacenamiento, utilización y reciclaje de agua en el Chaco Central. Loma Plata, PY: S.C.C CHORTITZER KOMITEE Ltda.-SAP-INTTAS. 68 p.

Israel, DW. 1987. Investigation of the role of phosphorus in symbiotic dinitrogen fixation. Plant Physiology 84:835-840.

Iqbal, A; Khalil, IA; Ateeq, N; Sayyar, MK. 2006. Nutritional quality of important food legumes. Food Chemistry 97(2): 331-335.

Jover, PL. 2003. Tecnología de producción y manejo poroto caupi. INTA. EEA Colonia Benítez, Chaco. AR. 32 p.

Kamara, CS. 1976. The effects of excess and deficient soil moisture on growth and yield of cowpea. Tropical Grain Legume bulletin. p. 6-4.

Langyintuo, AS; Lowenberg-DeBoerb, BJ; Fayec, M; Lambertb, D; Ibrod, G; Moussad, B; Kergnae, A; Kushwahaf, S; Musaf, S; Ntoukamg, G. 2003. Cowpea supply and demand in West and Central Africa, Field Crops Research 82(2-3)215-231.

López Guerra, CM; Bressani, R. 2008. Uso del cowpea (Vigna unguiculata) en mezclas con fríjol común (Phaseolus vulgaris) en el desarrollo de nuevos productos alimenticios. ALAN 58 (1):71-80.

MAG/DCEA (Ministerio de Agricultura y Ganadería, PY) DCEA (Dirección de Censos y Estadísticas Agropecuarias). 2013. Síntesis Estadísticas: producción agropecuaria 2012/2013. San Lorenzo, PY. 47 p.

Marchesini, A; Allievi, L; Comotti, E; Ferrari, A. 1988. A long-term effects of quality compost treatment on soil. Plant and Soil 106:253-261.

Miquilena, E; Higuera Moros; A. 2012. Evaluación del contenido de proteína, minerales y perfil de aminoácidos en harinas de Cajanus cajan, Vigna unguiculata y Vigna radiata para su uso en la alimentación humana. Revista Científica UDO Agrícola 12 (3): 730-740.

Oliveira, AP; Tavares Sobrinho, J; Nascimento, JT; Alves, AU; Albuquerque, IC; Bruno, GB. 2002. Avaliação de linhagens e cultivares de feijão-caupi, em Areia, PB. Horticultura Brasileira 20 (2):180-182.
Oliveira, IP; Dantas, JP. 1988. Nutrição mineral do caupi. In: O caupi no Brasil. Araujo, PPA; Watt, EE (Org). Brasília, DF: IITA; Embrapa, p. 405-430.

Rabery, SH; Dyck, L. 2011 Período de barbecho del suelo y crecimiento radicular del cártamo Carthamus tinctorius L. en función al contenido de agua en el Chaco Central. Investigación Agraria 13(1):13-18.

Santos, JF; Grangeiro, JIT; Brito, CH; Santos; MCCA. 2009. Produção e componentes produtivos de variedades de feijão caupi na microregião cariri paraibano. Engenharia Ambiental 6 (1):214-222.

Singh, BB. 2005. Cowpea (Vigna unguiculata [L.] Walp.). In.: Genetic Resources, Chromosome Engineering and Crop Improvement, Singh RJ, Jauhar PP (eds) v 1, CRC Press, Boca Raton, FL, USA, p. 117-162.

Silva, PSL; Montenegro, EE. 1997. Resposta do caupi à remoção de flores e vagens. Pesquisa Agropecuaria Brasileira 32 (7): 709-714.

Silva, AJ da, Uchoa, SCP, Alves, JMA, Lima, ACS, Santos, CSV dos, Oliveira, JMF de, Melo, VF. 2010. Resposta do feijão-caupi à doses e formas de aplicação de fósforo em Latossolo Amarelo do Estado de Roraima. Acta Amazônica 40 (1):31-36.

Silva, HAP da; Souza Galisa, P de; Silva Oliveira, RS da; Vidal, MS; Simões-Araújo, JL. 2012. Expressão gênica induzida por estresses abióticos em nódulos de feijão-caupi. Pesquisa Agropecuaria Brasileira 47(6): 797-807.

Timko, MP; Singh, BB. 2008. Cowpea, a multifunctional legume. In: Moore, PH , Ming, R (eds). Genomics of Tropical Crop Plants. p. 227-258.

Torres, SB; Oliveira, FN; Oliveira, RC; Fernandes, JB. 2008. Produtividade e morfologia de acessos de caupi, em Mossoró, RN. Horticultura Brasileira 26 (4):537-539.

Vural, H; Karasu, A. 2007. Variability studies in cowpea (Vigna unguiculata [L.] Walp.) varieties grown in Isparta, Turkey. Revista UDO Agrícola 7(1): 29-34. 2007.

Yamada, H., Kamata, H. 1989. Agricultural technological evaluation of organic farming and gardening I: effects of organic farming on yields of vegetables and soil physical and chemical properties. Bulletim of the Agricultural Research Institute of Kanagawa Prefecture, 130:1-13. 